JOURNAL OF SYNCHROTRON RADIATION

ISSN 1600-5775

Received 26 February 2016

Accepted 27 April 2016

Edited by J. F. van der Veen

Keywords: free-electron laser; undulator beamline design; simulations.

\section{Undulator beamline optimization with integrated chicanes for X-ray free-electron-laser facilities}

Eduard Prat,* Marco Calvi, Romain Ganter, Sven Reiche, Thomas Schietinger and Thomas Schmidt

Paul Scherrer Institut, CH-5232 Villigen PSI, Switzerland. *Correspondence e-mail: eduard.prat@psi.ch

An optimization of the undulator layout of X-ray free-electron-laser (FEL) facilities based on placing small chicanes between the undulator modules is presented. The installation of magnetic chicanes offers the following benefits with respect to state-of-the-art FEL facilities: reduction of the required undulator length to achieve FEL saturation, improvement of the longitudinal coherence of the FEL pulses, and the ability to produce shorter FEL pulses with higher power levels. Numerical simulations performed for the soft X-ray beamline of the SwissFEL facility show that optimizing the advantages of the layout requires shorter undulator modules than the standard ones. This proposal allows a very compact undulator beamline that produces fully coherent FEL pulses and it makes possible new kinds of experiments that require very short and high-power FEL pulses.

\section{Introduction}

X-ray free-electron lasers (FELs) are cutting-edge research tools with multiple scientific applications in fields such as physics, chemistry, material science and biology. State-of-theart FEL facilities are usually based on the SASE (self-amplified spontaneous emission) mechanism (Kondratenko \& Saldin, 1980; Bonifacio et al., 1984) and provide transversely coherent radiation pulses with peak powers of several tens of gigawatts and pulse durations of a few tens of femtoseconds and shorter (Emma et al., 2010; Ishikawa et al., 2012). Seeding techniques are used in different facilities to improve the longitudinal coherence and therefore the brightness of the SASE-FEL pulses (see, for example, Togashi et al., 2011; Amann et al., 2012; Allaria et al., 2012; Ackermann et al., 2013; Ratner et al., 2015). In addition to fully coherent FEL pulses, the scientific users of the FEL facilities strongly demand shorter and higher-power FEL pulses than the ones obtained at standard facilities (see, for instance, Capotondi et al., 2015).

The undulator beamline of a standard FEL facility consists of several undulator modules with a typical length of about 3-5 m each. The space between undulator modules, normally shorter than $1 \mathrm{~m}$, is used to place quadrupole magnets to focus the electron beam, phase shifters to control the phase between the electron and photon beams, dipole magnet correctors to steer the electron beam, and other components such as diagnostics.

In this work we present an optimization of the undulator layout of FEL facilities. We propose to add at every interundulator section a magnetic chicane consisting only of dipole magnets, i.e. a large phase shifter. Such chicanes have two physical effects: they delay the electron beam with respect to the produced FEL radiation and they introduce longitudinal 
dispersion $\left(R_{56}\right)$. In a chicane consisting of only dipole magnets the $R_{56}$ is approximately two times the electron beam delay. The maximum applied delay is up to a few femtoseconds; therefore such compact chicanes can be placed in the inter-undulator space of a typical FEL facility (see $\$ 6$ for more details).

Installing chicanes between the undulator modules has the following advantages: the $R_{56}$ of the chicanes can be used to reduce the undulator length required to reach saturation thanks to the optical klystron effect (Vinokurov \& Skrinsky, 1977), the delays can be employed to improve the longitudinal coherence of the SASE-FEL pulses without the need of any seeding technique (Thompson et al., 2010; Wu et al., 2013a; McNeil et al., 2013), and delaying the electrons opens up the possibility to achieve FEL pulses with much higher power and shorter durations than the ones produced in standard facilities (Prat \& Reiche, 2015; Prat et al., 2015). Our proposal represents a step forward in the design of FEL facilities: it allows a very compact design that offers fully coherent FEL pulses, and it can provide FEL pulses with unprecedented small durations and high powers, thereby creating new opportunities in FELbased sciences.

In the following sections we will describe in more detail the above-mentioned benefits of the new design and we will analyze the influence of the undulator module length on the performance of the scheme. Installing chicanes can offer other advantages such as the generation of phase-locked short pulse trains (Thompson \& McNeil, 2008); this option, however, requires additional hardware and will therefore not be further discussed here. We will see that maximizing the benefits of the chicanes, especially the improvement of the FEL brightness, requires shorter undulator modules than the standard ones. Hardware considerations of the new design are discussed in $\S 6$. We will use as an example the soft X-ray beamline of SwissFEL (Ganter, 2012), which is described in the next section, but the obtained results are of a general nature and are easily applicable to any other FEL facilities with similar beam parameters such as LCLS-II (Galayda, 2014).

\section{Example case: SwissFEL}

SwissFEL (Ganter, 2012) is an FEL facility under construction at the Paul Scherrer Institute. It will serve two beamlines: a hard X-ray beamline (Aramis), presently under construction, which will provide FEL radiation from 2017 with photon energies between 1.77 and $12.40 \mathrm{keV}$, and a soft X-ray beamline (Athos), currently in its design phase and expected to provide FEL light from 2020 with photon energies ranging between 0.18 and $1.77 \mathrm{keV}$.

The simulations shown in the following sections are performed using the code GENESIS 1.3 (Reiche, 1999) for the Athos case. Based on our design choices (Ganter, 2012) and our measurements at the SwissFEL Injector Test Facility (Prat et al., 2014), the electron parameters used in the simulations are the following, unless specified otherwise: the beam charge is $200 \mathrm{pC}$, the current has a flat profile with a peak value between 2 and $3 \mathrm{kA}$, corresponding to a total electron beam
Table 1

Typical simulation parameters.

\begin{tabular}{ll}
\hline Parameter & Value \\
\hline Electron beam charge & $200 \mathrm{pC}$ \\
Electron peak current & $2-3 \mathrm{kA}$ \\
Electron current profile & Flat \\
Electron pulse duration & $100-67 \mathrm{fs}$ \\
Electron energy & $3 \mathrm{GeV}$ \\
RMS uncorrelated energy spread & $350 \mathrm{keV}$ \\
Normalized transverse emittance & $300 \mathrm{~nm}$ \\
Undulator period & $40 \mathrm{~mm}$ \\
Photon energy & $1.24 \mathrm{keV}$ \\
\hline
\end{tabular}

duration between 100 and $67 \mathrm{fs}$, the energy is $3 \mathrm{GeV}$, the RMS uncorrelated energy spread is $350 \mathrm{keV}$, and the normalized emittance is $300 \mathrm{~nm}$. The undulator modules have a period of $40 \mathrm{~mm}$ and the photon energy is $1.24 \mathrm{keV}$, if not indicated differently. Table 1 shows the typical parameters used in the simulations presented in this paper.

Since Athos is in the design phase, the length of an undulator module is a parameter that still can be optimized for best performance. For the calculations presented here we will vary the undulator module length, thereby keeping the space between the modules fixed to $0.75 \mathrm{~m}$, which is sufficient to add a chicane to the existing components (see \$6). For each choice of undulator module length we focus the electron beam as much as possible. With shorter modules the electron beam can be more focused, which in general leads to a better FEL performance. For instance, the $\beta$-functions corresponding to module lengths of $1 \mathrm{~m}, 2 \mathrm{~m}$ and $4 \mathrm{~m}$ are $3 \mathrm{~m}, 5 \mathrm{~m}$ and $9 \mathrm{~m}$, respectively.

\section{Reduction of saturation length}

The undulator length required to achieve FEL saturation can be reduced by applying the optical klystron effect (Vinokurov \& Skrinsky, 1977), i.e. the generation of the microbunching during the FEL process can be accelerated with the $R_{56}$ of the dispersive sections between undulator modules. The optical klystron concept has been studied for SASE-FELs (Saldin $e t$ al., 2003; Ding et al., 2006) and has recently been demonstrated in a VUV FEL (Penco et al., 2015). A reduction of the saturation length implies a shorter undulator beamline or, alternatively, more space to increase the FEL power via tapering of the undulator field (Kroll et al., 1981).

The performance of the optical klystron configuration is very sensitive to the uncorrelated energy spread of the beam: the optical klystron effect in a given chicane is not effective when $R_{56} \sigma_{\delta}(2 \pi / \lambda)<1$ (Ding et al., 2006) (where $\sigma_{\delta}$ is the relative energy spread of the beam and $\lambda$ is the radiation wavelength). For our beam parameters at a radiation wavelength of $1 \mathrm{~nm}(1.24 \mathrm{keV})$, taking the maximum $R_{56}$ of $1 \mu \mathrm{m}$ (corresponding to a delay of $3.3 \mathrm{fs}$ ), the energy spread should be smaller than $480 \mathrm{keV}$. This condition is well fulfilled with our energy spread of $350 \mathrm{keV}$. The additional energy spread induced by incoherent synchrotron radiation in the chicanes turns out to be, for our parameters, insignificant compared with $350 \mathrm{keV}$. The uncorrelated energy spread will probably 
become a limitation for the implementation of the optical klystron effect for harder X-rays.

Fig. 1 displays the evolution of the FEL power along the undulator beamline with and without the optical klystron configuration for the Athos beamline with a module length of $4 \mathrm{~m}$. For the optical klystron case there is a magnetic chicane after each undulator module. The $R_{56}$ of each chicane is optimized to maximize the FEL power after the following undulator module. We consider delays between zero and $3.3 \mathrm{fs}$ in steps of $0.33 \mathrm{fs}$. The figure shows that the required length to achieve saturation decreases by about $30 \%$ (i.e. from about $32 \mathrm{~m}$ to about $22 \mathrm{~m}$ ) when harnessing the optical klystron effect.

Concerning the optimization of the module length, on the one hand a shorter module is preferable because the optical klystron effect can be applied in a more continuous and smooth manner. Moreover, a shorter module allows for stronger focusing and therefore smaller electron beam sizes, which are generally favorable for the FEL performance. On the other hand, a shorter module has the disadvantage of a lower filling ratio for the same inter-undulator spacing, i.e. the fraction of the beamline occupied by undulator modules is smaller. In addition, for a shorter module the mode mismatch between the undulator modules is worse: the transverse size of the FEL pulse is constant along the undulator module due to gain guiding but it increases within the drift space, no longer matching with the fundamental FEL eigenmode in the next undulator module, therefore causing an effective reduction of the field capable of coupling to the electron beam.

We have optimized the optical klystron configuration for eight different undulator module lengths: $0.5 \mathrm{~m}, 1 \mathrm{~m}, 1.5 \mathrm{~m}$, $2 \mathrm{~m}, 3 \mathrm{~m}, 4 \mathrm{~m}, 6 \mathrm{~m}$ and $8 \mathrm{~m}$. For all cases there is a chicane at every inter-undulator section and the focusing is adjusted to provide the minimum allowed $\beta$-function. Fig. 2 shows the FEL power along the undulator beamline using the optical klystron effect for the different module lengths. The shortest saturation length is around $20 \mathrm{~m}$ and is achieved for an

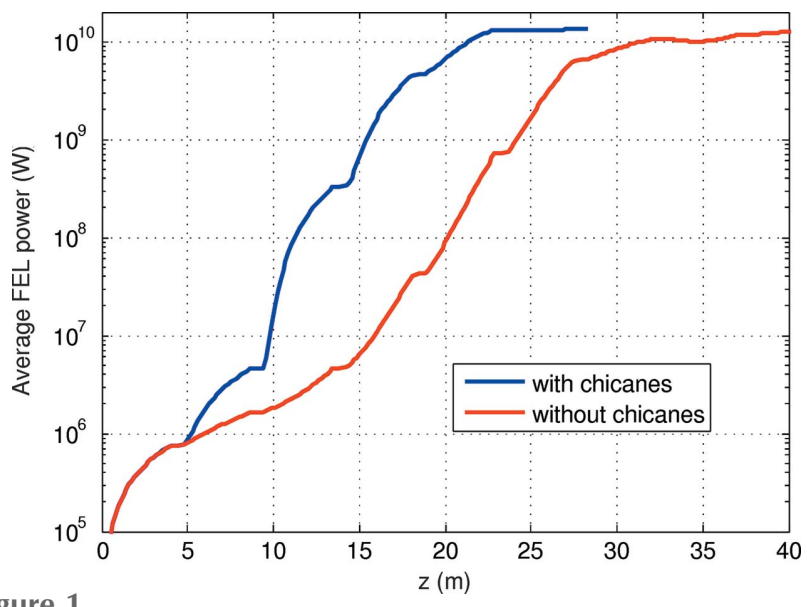

Figure 1

Power along the undulator beamline with and without optical klystron configuration for an undulator module length of $4 \mathrm{~m}$. There is a chicane in every inter-undulator space of $0.75 \mathrm{~m}$. The applied delays for the optical klystron are $2 \mathrm{fs}$ in the first two chicanes and $0.7 \mathrm{fs}$ in the third chicane, while the following chicanes are set to zero delay.

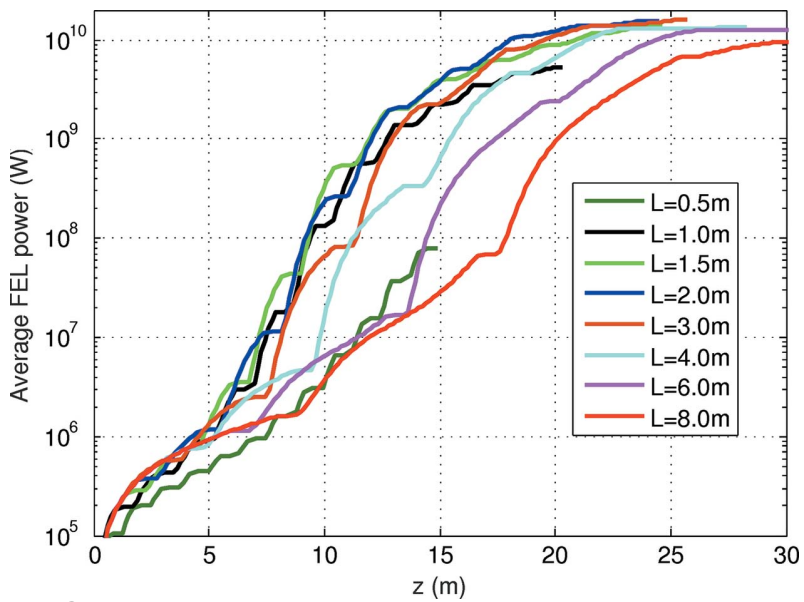

Figure 2

Power along the undulator beamline for optimum optical klystron configuration and for different undulator module lengths $L$.

undulator module length of around $2 \mathrm{~m}$. We note that without chicanes the optimum module length for minimum saturation length is about $3 \mathrm{~m}$, balancing focusing versus filling factor and mode mismatch. Therefore, the undulator module has to be shortened to benefit the most from the optical klystron effect.

\section{Improved coherence}

The natural bandwidth of the SASE-FEL pulses is of the order of the Pierce parameter $\rho$ (Bonifacio et al., 1984), with typical values for an FEL facility varying between $10^{-3}$ and $10^{-4}$. Various seeding methods can be used to decrease the bandwidth of the SASE-FEL pulses. One possibility is to use the self-seeding mechanism (Feldhaus et al., 1997; Saldin et al., 2001; Geloni et al., 2010): a monochromator is placed at a location in the undulator before the SASE-FEL reaches saturation levels, and the monochromatic signal is later used as a seed in a second amplification stage. Another option is to employ external lasers, either seeding directly with a highharmonic generation source (Ferray et al., 1988) or by using more complicated layouts with modulators and chicanes such as in the high-gain harmonic generation (Yu, 1991) or the echo-enabled harmonic generation (Stupakov, 2009) schemes. Self-seeding has been proven to work for hard and soft X-rays (Amann et al., 2012; Ratner et al., 2015), while laser-based seeding has a present limitation at a photon energy of around $0.3 \mathrm{keV}$ (Allaria et al., 2013). Surpassing this limitation seems very difficult due to a drop in the efficiency of external seeding sources and noise degradation problems (Saldin et al., 2002).

Instead of seeding, the longitudinal coherence of the SASEFEL pulses can be improved by delaying the electrons with respect to the photons between undulator modules, thus increasing the cooperation length between electrons and photons (Thompson et al., 2010; Wu et al., 2013a; McNeil et al., 2013); the cooperation length is the distance over which the phase of the signal is correlated. The delays required in this so-called high-brightness SASE (HB-SASE) scheme can be obtained with magnetic chicanes. Recently a proof-of-principle experiment of the HB-SASE scheme was successfully carried out in the X-ray regime (Wu et al., 2013b). 
Table 2

FEL bandwidth achieved with HB-SASE for different undulator module lengths compared with the standard SASE mode.

\begin{tabular}{ll}
\hline Module length $(\mathrm{m})$ & FWHM bandwidth $\left(\times 10^{-4}\right)$ \\
\hline 1 & $1.6 \pm 1.5$ \\
1.5 & $2.1 \pm 1.2$ \\
2 & $2.4 \pm 1.5$ \\
3 & $7.0 \pm 4.2$ \\
4 & $16.0 \pm 6.9$ \\
2 (SASE) & $19.4 \pm 10.3$ \\
\hline
\end{tabular}

Both with seeding and HB-SASE the FEL bandwidth can be reduced close to the Fourier limit. A jitter of the electron beam energy translates to an FEL pulse energy jitter in the seeding schemes but to a photon energy jitter in the HB-SASE case. The latter has the advantages of not requiring any laser or monochromator, and that it can be realised at any photon energy and repetition rate. In addition, the HB-SASE setup requires much less undulator length than self-seeding.

We have studied the performance of the HB-SASE mechanism for different module lengths of Athos. The electron peak current assumed in this case is $2 \mathrm{kA}$. The applied delays in the chicanes decrease monotonously along the undulator beamline, with an initial maximum delay of about 3 fs. Starting with larger values allows one to profit from the optical klystron effect, while ending with shorter delays prevents the electron beam from overbunching at the last stages. The FWHM bandwidth for the SASE case is around $2 \times 10^{-3}$. Table 2 shows the obtained results for module lengths of $1 \mathrm{~m}, 1.5 \mathrm{~m}, 2 \mathrm{~m}, 3 \mathrm{~m}$ and $4 \mathrm{~m}$. The total undulator length for all HB-SASE cases is about $20 \mathrm{~m}$, which is sufficient to achieve FEL saturation. For each module length we have taken the best result out of several delay configurations. The values shown in the table are averaged over ten random cases to take into account different seeds for the initial shot noise, and the error bars show the standard deviation over the ten cases. For modules longer than or equal to $4 \mathrm{~m}$ the bandwidth is not improved with respect to SASE. For 3 m modules the bandwidth is reduced by a factor of three, while for $2 \mathrm{~m}$ or less the bandwidth is improved by approximately a factor of ten. As expected, the best result is obtained for the shortest modules, since in this case the delays can be applied more regularly. Fig. 3 shows an average spectrum over ten seeds for SASE and HB-SASE for a module length of $1.5 \mathrm{~m}$, where the bandwidth reduction in the HB-SASE case can be clearly observed.

\section{Generation of short and high-power FEL pulses}

Two recent studies have proposed to use magnetic chicanes between undulator modules to produce shorter and highpower FEL pulses. Both schemes are based on superradiance (Bonifacio et al., 1990, 1991), a regime with quadratic growth of the radiation power as a function of the undulator length and a shortening of the pulse length while it slips into fresh (unspoiled) regions of the electron bunch. In the first proposal (Prat \& Reiche, 2015), a multiple-slotted foil determines

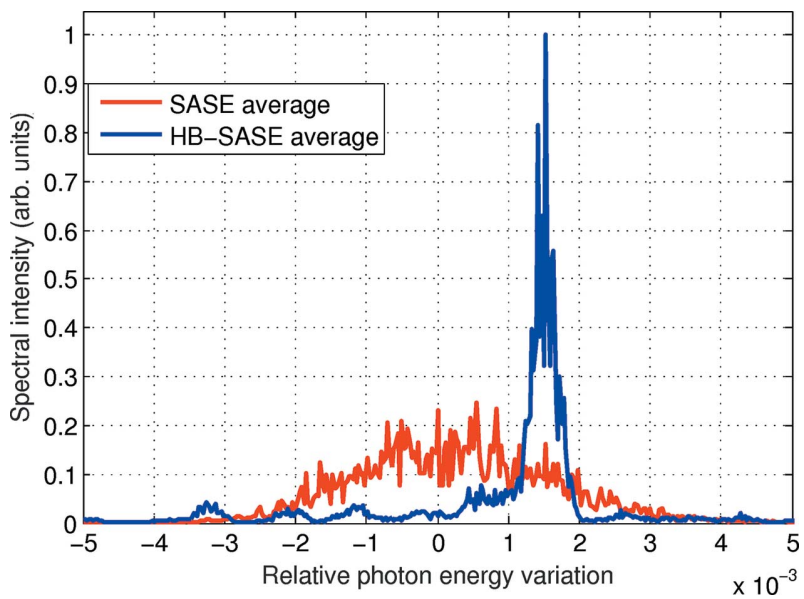

Figure 3

FEL average spectrum for SASE and HB-SASE for an undulator module length of $1.5 \mathrm{~m}$. The zero of the horizontal axis corresponds to a photon energy of $1.2375 \mathrm{keV}$. With the HB-SASE the bandwidth is reduced by about a factor of ten. The comparison is made for similar final FEL energies in both cases, corresponding to an undulator beamline of $20 \mathrm{~m}$ for the HB-SASE configuration and of about $30 \mathrm{~m}$ for the SASE case.

several unspoiled regions of the electron beam, which will produce several FEL pulses in the first section of the undulator beamline. Then, by suitably delaying the electron beam with magnetic chicanes after some undulator modules, only the first of the initial FEL pulses will be enhanced by all the other unspoiled regions of the electron beam. The separation between the slots in the foil must be uneven to prevent the growth of the other unwanted FEL pulses. The second method (Prat et al., 2015) suggests inducing a transverse tilt, i.e. a correlation between transverse and longitudinal (or time) coordinates, to the electron bunch. The tilt can be generated in different ways using standard components of a typical FEL facility. In the first part of the undulator beamline, only the tail of the electron beam will have an aligned trajectory and therefore generate significant radiation. After that, by properly delaying the electron beam with chicanes and correcting its trajectory between certain undulator modules, the whole electron bunch can contribute to amplify a short FEL pulse. This second proposal is more efficient since all the electrons can contribute to the FEL process, and more flexible since by tuning the tilt amplitude one can minimize the pulse duration or maximize the FEL pulse energy.

We have simulated the generation of short and high-power FEL pulses using a transverse tilt for the soft X-ray beamline Athos; we refer to Prat \& Reiche (2015) and Prat et al. (2015) for simulations for the hard X-ray beamline of SwissFEL. The tilt is defined with its amplitudes in offset $\mathrm{d} x / \mathrm{d} s$ and angle $\mathrm{d} x^{\prime} / \mathrm{d} s$, where $x$ is the transverse offset, $x^{\prime}$ is the transverse angle, and $s$ is the longitudinal coordinate along the bunch. We assume a peak current of $6 \mathrm{kA}$ corresponding to a total electron beam duration of $33 \mathrm{fs}$ for $200 \mathrm{pC}$, and a photon energy of $0.62 \mathrm{keV}$. A higher current than the design one is justified in this configuration because the longitudinal wakefields of the undulator only have to be compensated locally at the slices that contribute to the amplification of the short FEL pulse, in contrast to the conventional SASE configuration, 
where the longitudinal wakefields need to be corrected rather globally to allow the full bunch to drive the FEL process. We have performed the simulations for three different undulator module lengths $(1 \mathrm{~m}, 2 \mathrm{~m}$ and $4 \mathrm{~m})$ and for two different transverse tilt amplitudes only in offset at the undulator entrance (offset variation along the bunch of $1.5 \mathrm{~mm}$ and $3 \mathrm{~mm}$ ). The maximum tilt amplitude is determined by the dimensions of the undulator vacuum chamber; in our case the undulator half-gap is $3 \mathrm{~mm}$, which is sufficient for the considered tilt amplitudes. We use eight undulator sections, consisting of one or more undulator modules each, with seven chicanes in between. For a module length of $4 \mathrm{~m}$, the first undulator section consists of four undulator modules and the rest of the sections contain one undulator module each. When the modules are $2 \mathrm{~m}$ long, we employ six modules in the first undulator section and two modules for all the other sections. Finally, for the case of $1 \mathrm{~m}$ modules, 12 modules are used in the first undulator section, and four in all the following ones. The length of the first undulator section varies for different module lengths because the undulator length to reach saturation is reduced for shorter modules due to stronger beam focusing in this case. In total, 11, 20 and 40 undulator modules are employed with module lengths of $4 \mathrm{~m}, 2 \mathrm{~m}$ and $1 \mathrm{~m}$, respectively. The total undulator beamline length amounts to approximately $50 \mathrm{~m}$ for module lengths of $4 \mathrm{~m}$ or $2 \mathrm{~m}$, and about $70 \mathrm{~m}$ in the case of $1 \mathrm{~m}$ modules. Fig. 4 shows the three layouts for the different modules lengths. The delay of each chicane is calculated as the total bunch length divided by the number of sections (8) less the slippage length along the upstream undulator section, which in our case is about 3 fs for all configurations.

For each of the cases we have run five simulations using different seeds for the generation of the electrons' shot noise. We have not considered transverse wakefield effects related to the beam tilt. The performance of the scheme, however, only relies on the on-axis part of the bunch, therefore any kicks induced by transverse wakefields in previous stages can be compensated locally. Table 3 shows the results for all six

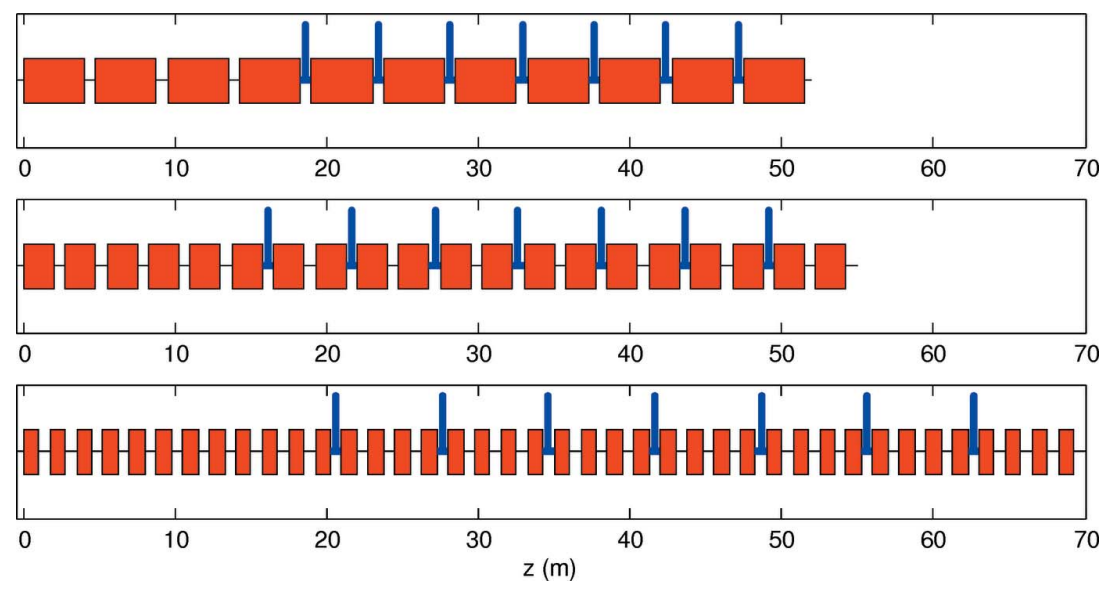

Figure 4

Layout drawings for the generation of the short and high-power FEL pulses for different module lengths: $4 \mathrm{~m}$ (top), $2 \mathrm{~m}$ (middle) and $1 \mathrm{~m}$ (bottom). The undulator modules are indicated with red rectangles, the magnetic chicanes with blue lines.
Table 3

Generation of short and high-power FEL pulses: results for three undulator module lengths $(L)$ and two tilt amplitudes in offset.

\begin{tabular}{llll}
\hline Configuration & $\begin{array}{l}\text { FEL peak } \\
\text { power (TW) }\end{array}$ & $\begin{array}{l}\text { FEL pulse } \\
\text { energy (mJ) }\end{array}$ & $\begin{array}{l}\text { FWHM pulse } \\
\text { duration (fs) }\end{array}$ \\
\hline$L=4 \mathrm{~m}$, tilt $1.5 \mathrm{~mm}$ & $1.02 \pm 0.07$ & $2.21 \pm 0.14$ & $3.21 \pm 0.42$ \\
$L=4 \mathrm{~m}$, tilt $3.0 \mathrm{~mm}$ & $1.40 \pm 0.19$ & $1.81 \pm 0.19$ & $1.92 \pm 0.90$ \\
$L=2 \mathrm{~m}$, tilt $1.5 \mathrm{~mm}$ & $1.62 \pm 0.58$ & $1.01 \pm 0.24$ & $0.46 \pm 0.26$ \\
$L=2 \mathrm{~m}$, tilt $3.0 \mathrm{~mm}$ & $1.48 \pm 0.20$ & $0.52 \pm 0.05$ & $0.30 \pm 0.01$ \\
$L=1 \mathrm{~m}$, tilt $1.5 \mathrm{~mm}$ & $1.52 \pm 0.06$ & $0.64 \pm 0.02$ & $0.30 \pm 0.01$ \\
$L=1 \mathrm{~m}$, tilt $3.0 \mathrm{~mm}$ & $0.46 \pm 0.16$ & $0.18 \pm 0.06$ & $0.34 \pm 0.01$ \\
\hline
\end{tabular}

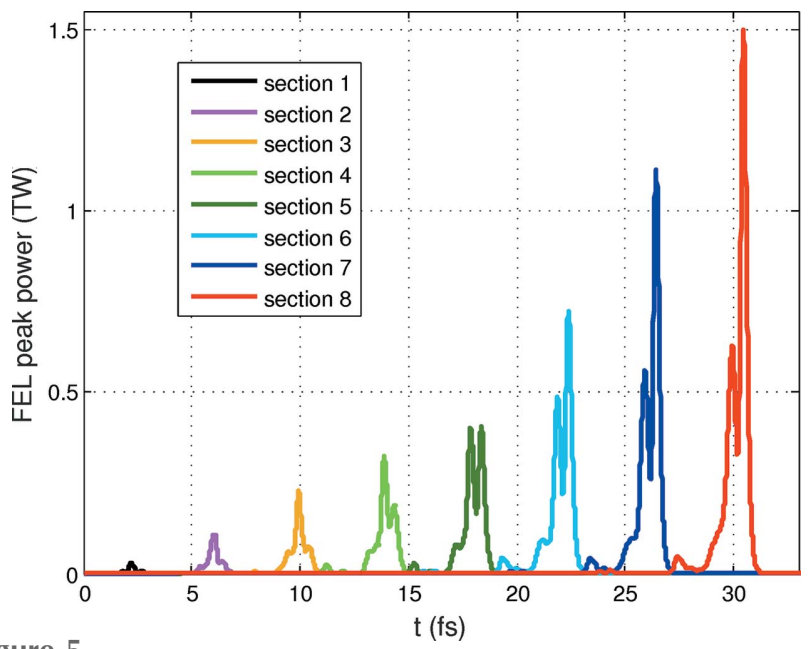

Figure 5

FEL peak power along the time coordinate of the electron bunch after each undulator section for a module length of $2 \mathrm{~m}$ and a tilt in offset along the bunch of $1.5 \mathrm{~mm}$.

simulated configurations in terms of FEL peak power, pulse energy and pulse duration. The error bars indicate the standard deviation of the results over the five simulation seeds. The worst FEL performance occurs for a module length of $1 \mathrm{~m}$ and an initial tilt in offset of $3 \mathrm{~mm}$. This is because the effect of reducing the FEL pulse length by increasing the tilt or reducing the betatron function is limited by the cooperation length of the FEL process. If the cooperation length is such that the field would slip into parts of the bunch where the betatron oscillation suppresses the FEL amplification, the whole process breaks down, as is the case for a module length of $1 \mathrm{~m}$ and a tilt in offset of $3 \mathrm{~mm}$. For all the other cases, a peak power above $1 \mathrm{TW}$ is achieved. The shortest FEL pulse is about $0.3 \mathrm{fs}$, which corresponds to a single radiation spike. The FEL power profile after each undulator section for one random seed for the case of a module length of $2 \mathrm{~m}$ and a tilt in offset of $1.5 \mathrm{~mm}$ is shown in Fig. 5. As is evident from Table 3, when the module lengths are $4 \mathrm{~m}$ and $2 \mathrm{~m}$, a larger tilt generates a shorter FEL pulse but with less FEL pulse energy, while a smaller 
tilt produces longer but more energetic FEL pulses; this effect is discussed in more detail by Prat et al. (2015). This result is not observed for a module length of $1 \mathrm{~m}$ since for this case the FEL performance is simply degraded when going to larger tilts, as explained above.

Shorter modules are generally preferable. First of all, the tilt required for a given performance is reduced when using shorter modules, because in this case the focusing can be stronger and, therefore, an equivalent effect in terms of FEL disruption can be achieved with smaller tilts. In our case, for an initial offset of $3 \mathrm{~mm}$ the FEL pulse is shortened to about 2 fs for a module of $4 \mathrm{~m}$, while for $2 \mathrm{~m}$ modules an initial offset of $1.5 \mathrm{~mm}$ is sufficient to reduce the pulse duration to about $0.5 \mathrm{fs}$. Equivalently, a tilt offset of $3 \mathrm{~mm}$ is required to achieve a pulse duration of $0.3 \mathrm{fs}$ for a module length of $2 \mathrm{~m}$, while for $1 \mathrm{~m}$ module length a tilt offset of $1.5 \mathrm{~mm}$ is sufficient to obtain the same pulse duration. In addition, the smaller beam sizes achievable with shorter modules in general improve the FEL performance; in our simulations the FEL peak power is larger for the cases where the module is $2 \mathrm{~m}$ and $1 \mathrm{~m}$ long (except for the problematic case with the strongest tilt and $1 \mathrm{~m}$ modules). Moreover, with shorter modules the delays could be applied more often, which could potentially lead to even higher FEL peak powers. Finally, with shorter modules, it is easier to prevent a radiation spike from slipping over the location of the next spike, which would saturate the FEL power increase; this is particularly important for smaller photon energies. Going to shorter modules has, however, the disadvantage that, due to the lower filling factor the required beamline length to achieve a given performance is increased: for our case, the undulator beamline increased from about $50 \mathrm{~m}$ to $70 \mathrm{~m}$ when going to a module length of $1 \mathrm{~m}$.

\section{Hardware considerations}

\subsection{Inter-undulator sections}

In our proposal the space between the undulator modules is equipped with a magnetic chicane, a quadrupole magnet to focus the beam, dipole corrector magnets to tune the electron beam trajectory, and other components such as diagnostics and vacuum valves.

We propose to build dispersive chicanes with only dipole magnets. In this way, the delay and the longitudinal dispersion are coupled; the $R_{56}$ is approximately equal to twice the beam delay. Another possibility would be to use isochronous chicanes that delay the electrons but have no longitudinal dispersion, $R_{56}=0$, and therefore do not affect the microbunching of the electron beam, as proposed for instance in the original HB-SASE study (Thompson et al., 2010). Such chicanes would require quadrupole magnets to close the dispersion and would occupy much more space than in our proposal. As mentioned earlier, our design with its decremental sequence of delays takes advantage of the microbunching generation in the first undulator stages (optical klystron) while avoiding significant overbunching in the latter stages. Therefore, there is no loss in performance and the design becomes much easier and compact.

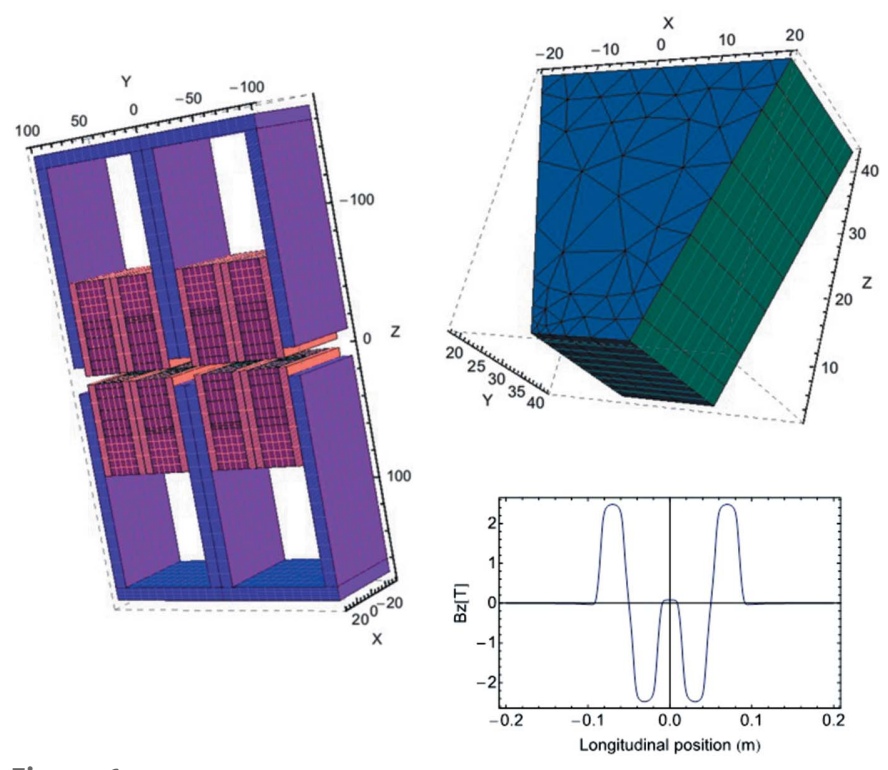

Figure 6

Design of the chicane. Simulation domain of the permanent-magnet dipole chicane (left). The pole is embedded in the permanent magnet (right-top). The right-bottom graph shows the magnetic field profile for the minimum gap of $6.5 \mathrm{~mm}$, which corresponds to a maximum delay of $5 \mathrm{fs}$ for an electron beam of $3 \mathrm{GeV}$.

To be as compact as possible the chicane should consist of permanent magnets, as considered for instance for the phase shifters of the European XFEL project (Lu et al., 2009). Fig. 6 shows the design of a possible permanent-magnet chicane for SwissFEL calculated with the code RADIA (Chubar et al., 1998). The chicane consists of four pairs of magnets (the four dipoles), separated by an adjustable gap, and has a total length of $0.2 \mathrm{~m}$; this is sufficiently small to guarantee that the chicane and the rest of the components can be placed in the $0.75 \mathrm{~m}$ assumed in the simulations. At the center of each magnet there is a trapezoidally shaped pole to enhance the field. At the minimum gap of $6.5 \mathrm{~mm}$, an electron bunch with an energy of $3 \mathrm{GeV}$ can be delayed by $5 \mathrm{fs}$, which is more than sufficient for the purposes of our design. When the gap has a large opening, the chicane can be used as a normal phase shifter as required when the FEL is operated in the normal SASE mode. Each half of the chicane can be motorized separately, which allows the additional generation of a transverse offset, if one half is weaker than the other. This feature will be used to produce short and high-power FEL pulses. In addition to the chicane, we plan to install one dipole corrector magnet to have independent control of the offset and angle of the electron beam trajectory.

\subsection{Undulator modules}

Achieving the optimum performance in our scheme calls for undulator modules that are shorter than in today's standard facilities. This is clearly borne out in Fig. 7, which gives a graphical summary of the quantitative aspects of our investigations into the three operation modes considered in this work. 

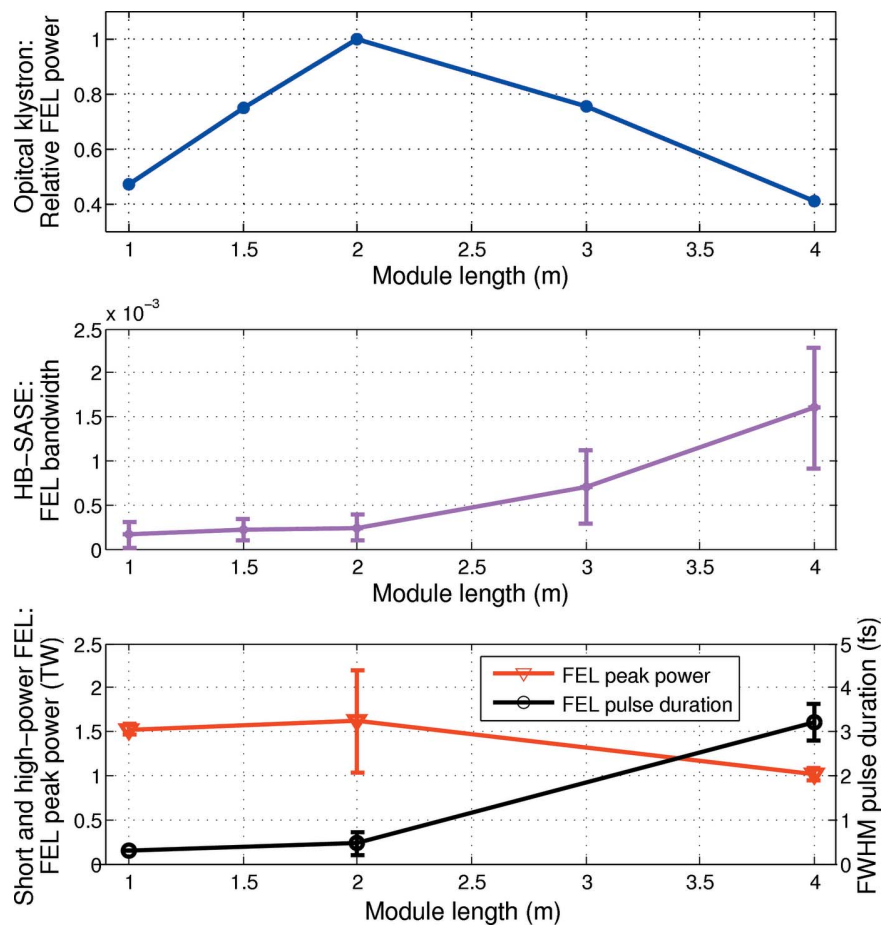

Figure 7

Summary of FEL performance as a function of the module length. Top: relative FEL power obtained when using the optical klystron effect (see Fig. 2; the values shown here are averaged between $z=15 \mathrm{~m}$ and $z=$ $20 \mathrm{~m}$ ). Middle: FEL bandwidth obtained with HB-SASE (see Table 2). Bottom: FEL peak power and pulse duration when using a tilt in offset of $1.5 \mathrm{~mm}$ (see Table 3).

Despite the lower filling ratio and the poorer mode mismatch, going to shorter modules is preferable to maximize the optical klystron effects: for the case of Athos, a 2 m-long module would give the minimum saturation length. In addition, the shortening of the module length is required to have a significant improvement of the brightness: for our case, no improvement in the brightness is observed for a module length of $4 \mathrm{~m}$; with a module of $3 \mathrm{~m}$ the brightness improvement is only a factor of three, whereas for modules of $2 \mathrm{~m}$ or shorter the improvement amounts to a factor of around ten. Finally, the generation of short and high-power FEL pulses is more efficient for shorter modules: the required tilt amplitude is smaller to obtain a certain pulse duration, the FEL performance improves since the beam sizes can be smaller and the delays can be applied more often.

Based on our findings the undulators in the current Athos design have a total length of $2 \mathrm{~m}$. The modules will be Apple devices (Clarke, 2004; Schmidt et al., 2015) with a period length of $40 \mathrm{~mm}$. The magnets will be made of samariumcobalt. We chose samarium-cobalt because in comparison with other materials (e.g. neodymium) it offers a low permeability, it is very resistant against radiation, and its remanence is almost insensitive to temperature variations. The undulator gap will vary from 3 to $25 \mathrm{~mm}$, which corresponds to a range for the undulator field parameter $K$ of between 3.8 and 1 . Considering that the electron beam energy for Athos can vary between 2.5 and $3.5 \mathrm{GeV}$, the tuning range of the undulator field is sufficient to cover the photon energy range from 0.18 to $1.77 \mathrm{keV}$.

\section{Conclusion}

We have presented an optimization of the undulator beamlines of FEL facilities based on installing chicanes in the space between the undulator modules. We have demonstrated with numerical simulations carried out for the Athos soft X-ray beamline of SwissFEL the merits of the integrated chicanes: the saturation length can be reduced by about $30 \%$, the brightness can be improved by about a factor of ten without the need of any seeding technique, and it is possible to generate short and high-power FEL pulses. To fully use the merits of the integrated chicanes, especially for the generation of longitudinally coherent FEL pulses, the length of an undulator module needs to be shorter than in the standard facilities; for our case, the module length should be about $2 \mathrm{~m}$. We note that going to shorter undulator modules has additional advantages, such as the possibility to generate FEL radiation at the harmonics of the radiation $(\mathrm{McNeil}$ et al., 2006). Our scheme offers the possibility to have a very compact undulator beamline that generates almost fully coherent FEL pulses and it opens the door for new types of experiments that require very short and high-power FEL pulses. For the Athos case, fully coherent FEL pulses can be obtained within $20 \mathrm{~m}$ of the undulator beamline, and TW pulses shorter than $1 \mathrm{fs}$ can be generated in about $50 \mathrm{~m}$.

\section{Acknowledgements}

We are indebted to Hans Braun for fruitful discussions that helped to improve the content of the document. We thank Terry Garvey for the careful proofreading of the manuscript.

\section{References}

Ackermann, S. et al. (2013). Phys. Rev. Lett. 111, 114801.

Allaria, E. et al. (2012). Nat. Photon. 6, 699-704.

Allaria, E. et al. (2013). Nat. Photon. 7, 913-918.

Amann, J. et al. (2012). Nat. Photon. 6, 693-698.

Bonifacio, R., De Salvo Souza, L., Pierini, P. \& Piovella, N. (1990). Nucl. Instrum. Methods Phys. Res. A, 296, 358-367.

Bonifacio, R., Pellegrini, C. \& Narducci, L. M. (1984). Opt. Commun. 50, 373-378.

Bonifacio, R., Piovella, N. \& McNeil, B. W. J. (1991). Phys. Rev. A, 44, R3441-R3444.

Capotondi, F., Dell'Angela, M., Malvestuto, M. \& Parmigiani, F. (2015). Synchrotron Radiation: Basics, Methods and Applications, ch. 30. Berlin: Springer.

Chubar, O., Elleaume, P. \& Chavanne, J. (1998). J. Synchrotron Rad. 5, 481-484.

Clarke, J. A. (2004). The Science and Technology of Undulators and Wigglers. Oxford University Press.

Ding, Y., Emma, P., Huang, Z. \& Kumar, V. (2006). Phys. Rev. ST Accel. Beams, 9, 070702.

Emma, P. et al. (2010). Nat. Photon. 4, 641-647.

Feldhaus, J., Saldin, E. L., Schneider, J. R., Schneidmiller, E. A. \& Yurkov, M. V. (1997). Opt. Commun. 140, 341-352.

Ferray, M., L'Huillier, A., Li, X. F., Lompre, L. A., Mainfray, G. \& Manus, C. (1988). J. Phys. B, 21, L31-L35. 
Galayda, J. N. (2014). Proceedings of the 5th International Particle Accelerator Conference, pp. 935-937.

Ganter, R. (2012). SwissFEL conceptual design report. Report 10-04. Paul Scherrer Institute, Switzerland.

Geloni, G. V. K. \& Saldin, E. (2010). A simple method for controlling the line width of SASE X-ray FELs. Report 10-053. Deutsches Elektronen-Synchrotron, Hamburg, Germany.

Ishikawa, T. et al. (2012). Nat. Photon. 6, 540-544.

Kondratenko, A. M. \& Saldin, E. L. (1980). Part. Accel. 10, 207-216.

Kroll, N. M., Morton, P. L. \& Rosenbluth, M. N. (1981). IEEE J. Quantum Electron. 17, 1436-1468.

Lu, H. H., Li, Y. \& Pflueger, J. (2009). Nucl. Instrum. Methods Phys. Res. A, 605, 399-408.

McNeil, B. W. J., Robb, G. R. M., Poole, M. W. \& Thompson, N. R. (2006). Phys. Rev. Lett. 96, 084801.

McNeil, B. W. J., Thompson, N. R. \& Dunning, D. J. (2013). Phys. Rev. Lett. 110, 134802.

Penco, G., Allaria, E., De Ninno, G., Ferrari, E. \& Giannessi, L. (2015). Phys. Rev. Lett. 114, 013901.

Prat, E., Aiba, M., Bettoni, S., Beutner, B., Reiche, S. \& Schietinger, T. (2014). Phys. Rev. ST Accel. Beams, 17, 104401.

Prat, E., Löhl, F. \& Reiche, S. (2015). Phys. Rev. ST Accel. Beams, 18, 100701.

Prat, E. \& Reiche, S. (2015). Phys. Rev. Lett. 114, 244801.

Ratner, D. et al. (2015). Phys. Rev. Lett. 114, 054801.

Reiche, S. (1999). Nucl. Instrum. Methods Phys. Res. A, 429, $243-$ 248.
Saldin, E. L., Schneidmiller, E. A., Shvyd'ko, Y. V. \& Yurkov, M. V. (2001). Nucl. Instrum. Methods Phys. Res. A, 475, 357-362.

Saldin, E. L., Schneidmiller, E. A. \& Yurkov, M. V. (2002). Opt. Commun. 202, 169-187.

Saldin, E. L., Schneidmiller, E. A. \& Yurkov, M. V. (2003). The Free Electron Laser Klystron Amplifier Concept. Report 03-108. Deutsches Elektronen-Synchrotron, Hamburg, Germany.

Schmidt, T., Anghel, A., Böhler, P., Brügger, M., Calvi, M., Danner, S., Huber, P., Keller, A. \& Locher, M. (2015). Proceedings of the 36th International Free-Electron Laser Conference, pp. 116-120.

Stupakov, G. (2009). Phys. Rev. Lett. 102, 074801.

Thompson, N. R., Dunning, D. J. \& McNeil, B. W. J. (2010). Proceedings of the 1st International Particle Accelerator Conference, pp. 2257-2259.

Thompson, N. R. \& McNeil, B. W. J. (2008). Phys. Rev. Lett. 100, 203901.

Togashi, T. et al. (2011). Opt. Express, 19, 317-324.

Vinokurov, N. A. \& Skrinsky, A. N. (1977). Power limitation in storage ring optical klystron. Report BINP 77-59. Budker Institute for Nuclear Physics, Novosibirsk, Russia.

Wu, J., Marinelli, A. \& Pellegrini, C. (2013a). Proceedings of the 34th International Free-Electron Laser Conference, pp. 237-240.

Wu, J., Pellegrini, C., Marinelli, A., Nuhn, H.-D., Decker, F.-J., Loos, H., Lutman, A., Ratner, D., Feng, Y., Krzywinski, J., et al. (2013b). Proceedings of the 4th International Particle Accelerator Conference, pp. 2068-2070.

Yu, L. H. (1991). Phys. Rev. A, 44, 5178-5193. 